\title{
Anodizing color coded anodized Ti6Al4V medical devices for increasing bone cell functions
}

\author{
This article was published in the following Dove Press journal: \\ International Journal of Nanomedicine \\ 3 January 2013 \\ Number of times this article has been viewed
}

\author{
Alexandra P Ross \\ Thomas J Webster \\ School of Engineering and \\ Department of Orthopedics, Brown \\ University, Providence, RI, USA
}

\begin{abstract}
Current titanium-based implants are often anodized in sulfuric acid $\left(\mathrm{H}_{2} \mathrm{SO}_{4}\right)$ for color coding purposes. However, a crucial parameter in selecting the material for an orthopedic implant is the degree to which it will integrate into the surrounding bone. Loosening at the bone-implant interface can cause catastrophic failure when motion occurs between the implant and the surrounding bone. Recently, a different anodization process using hydrofluoric acid has been shown to increase bone growth on commercially pure titanium and titanium alloys through the creation of nanotubes. The objective of this study was to compare, for the first time, the influence of anodizing a titanium alloy medical device in sulfuric acid for color coding purposes, as is done in the orthopedic implant industry, followed by anodizing the device in hydrofluoric acid to implement nanotubes. Specifically, Ti6A14V model implant samples were anodized first with sulfuric acid to create color-coding features, and then with hydrofluoric acid to implement surface features to enhance osteoblast functions. The material surfaces were characterized by visual inspection, scanning electron microscopy, contact angle measurements, and energy dispersive spectroscopy. Human osteoblasts were seeded onto the samples for a series of time points and were measured for adhesion and proliferation. After 1 and 2 weeks, the levels of alkaline phosphatase activity and calcium deposition were measured to assess the long-term differentiation of osteoblasts into the calcium depositing cells. The results showed that anodizing in hydrofluoric acid after anodizing in sulfuric acid partially retains color coding and creates unique surface features to increase osteoblast adhesion, proliferation, alkaline phosphatase activity, and calcium deposition. In this manner, this study provides a viable method to anodize an already color coded, anodized titanium alloy to potentially increase bone growth for numerous implant applications.
\end{abstract}

Keywords: nanotechnology, titanium, osteoblasts, anodization

\section{Introduction}

Of the estimated 200,000 spinal fusions that are performed each year, there is a failure rate of between $7 \%-35 \%{ }^{1}$ similar statistics could be reported for other orthopedic anatomical locations. Revision surgery to correct failures can be painful, expensive, and risky. Of several implant chemistries, titanium has been commonly used in the medical device industry due to its properties of being lightweight, having a low elastic modulus, being of high strength, and being corrosion resistant. ${ }^{2}$ The titanium alloy, Ti6Al4V, is frequently selected over other grades of titanium due to its increased mechanical properties. ${ }^{2}$ Of the many criteria that must be fulfilled, successful implantation requires that the surface of the Ti6Al4V integrates with the adjacent bone. ${ }^{2}$ One common cause of implant failure is the lack of bony fusion surrounding the
Correspondence: Thomas J Webster School of Engineering, Brown University, Providence, RI 02912, USA

Tel +14015233802

Fax +14015239107

Email thomas_webster@brown.edu 
implantation site. Loosening at the bone-implant interface can lead to failure when relative motion occurs between the implant and the surrounding bone. ${ }^{3,4}$ Such micromotion (which can be caused by extensive fibrous tissue formation) further prevents osseointegration. ${ }^{4}$

Current orthopedic industry standards use anodization for the purpose of color coding in order to improve the efficiency and accuracy of inserting titanium alloy devices in the operating room. Such titanium alloy surfaces are first bead blasted to remove machining defects on the surface, and they then undergo anodization in sulfuric acid for passivation and color coding (such as to label different implant sizes). Recently, however, there have been many advances in using a hydrofluoric acid-based anodization process to create nanotubes on titanium-based implants to improve bone growth. ${ }^{5}$ Such studies have demonstrated increased in vitro and in vivo bone cell responses, decreased bacteria functions, and inhibited inflammatory cell responses on titanium anodized in hydrofluoric acid compared to nontreated titanium samples. ${ }^{5-9}$ Research has also indicated that increased bone cell responses resulted in anodized nanotubular titanium due to its greater hydrophilicity, which promoted the adsorption of vitronectin, a well known osteoblast adhesive protein. ${ }^{5-9}$ Despite such promise in the use of hydrofluoric acid to improve bone growth, no studies (to the best of the authors' knowledge) have anodized current color coded titaniumbased spinal implants in hydrofluoric acid to determine bone growth ability, which would clearly represent a more accurate commercial scenario. For these reasons, the goal of this study was to compare and combine these two anodization practices to implement nanometer surface features, which can improve bone cell responses while preserving color coding functions.

\section{Materials and methods Sample preparation}

All of the titanium samples used in this study were machined from a $12.2 \mathrm{~mm}$ bar stock of Ti6Al4V ELI (Symmetry Medical Inc, New Bedford, MA, USA) using a lathe into disks $2 \mathrm{~mm}$ in thickness and $10 \mathrm{~mm}$ in diameter. After machining, all samples were passivated in 30\% nitric acid (DePuy Spine, Inc, Raynham, MA, USA) at $49^{\circ} \mathrm{C}$ for 20 minutes to remove any dirt or free iron introduced from the machining process. One group of samples (Group A) was ground with a polished belt (120 Grit Belt, Massasoit Tool; Massasoit, Attleboro, MA, USA) to produce a mechanical satin finish. All other samples (including Group B) were bead blasted with glass beads $125-250 \mu \mathrm{m}$ in diameter (US Standard number 10) per MIL G-9954A in order to produce a matte finish on the surface. Prior to anodizing or etching, samples were soaked in $70 \%$ ethanol for 10 minutes and they were then soaked in deionized water (Milli-Q Advantage Ultrapure Water Purification System; EMD Millipore, Billerica, MA, USA) for another 10 minutes. A subset of samples (Group C) was sent for anodization in sulfuric acid via a commercial process (Electropolishing Systems, Inc, Plymouth, MA, USA) to produce a green/yellow hue that is typically used for color coding purposes. The samples in Group $\mathrm{C}$ were anodized in $0.8 \%$ sulfuric acid for 90 seconds at $96 \mathrm{~V}$. After sulfuric acid anodization, a subset of samples (Group D) was anodized again using 1.5 weight percent ( $w \mathrm{t} \%$ ) hydrofluoric acid (Fluka; Sigma-Aldrich, St Louis, MO, USA). This was carried out by attaching each titanium alloy sample to a voltage source through copper wires.

A square of platinum mesh (Alfa Aesar, Ward Hill, MA, USA) was positioned $2 \mathrm{~cm}$ away from the surface of the titanium samples to act as a cathode. A constant voltage of $20 \mathrm{~V}$ was applied for 5 minutes. The final group of samples (Group E) was etched in $1.5 \mathrm{wt} \%$ of hydrofluoric acid for 5 minutes. After treatment with hydrofluoric acid, all samples were sonicated in $70 \%$ ethanol for 10 minutes, then in deionized water for 10 minutes, and then air-dried for an additional 10 minutes. Table 1 lists the processing treatments for each group tested in this study.

\section{Surface characterization}

The surface morphology of the samples was assessed using a JEOL JSM-5900LV scanning electron microscope (JEOL, Tokyo, Japan). Images were taken using a voltage of $15 \mathrm{kV}$ to capture surface features at varying magnification levels for each sample. No gold sputtering was used for this process. The chemical composition of each group of samples was determined through energy dispersive spectroscopy using an $\mathrm{INCA}^{\circledR}$ (Oxford Instruments, Abingdon, United Kingdom) detector in conjunction with the same scanning electron microscope used for scanning electron microscopy images. Contact angles were measured using a drop shape analysis

Table I Preparation of Ti6Al4V of interest to this study

\begin{tabular}{lll}
\hline Sample & Initial surface finish & Treatment \\
\hline Group A & Polished & None \\
Group B & Bead blasted & None \\
Group C & Bead blasted & Anodization in sulfuric acid \\
Group D & Bead blasted & $\begin{array}{l}\text { Anodization in sulfuric acid followed } \\
\text { by anodization in hydrofluoric acid }\end{array}$ \\
Group E & Bead blasted & $\begin{array}{l}\text { Anodization in sulfuric acid followed } \\
\text { by etching in hydrofluoric acid }\end{array}$ \\
\hline
\end{tabular}


system (EasyDrop; KRÜSS GmbH, Hamburg, Germany). The contact angle from $5 \mu \mathrm{L}$ double distilled water droplets was measured 15 seconds after the drop contacted the titanium alloy surface. Three samples from each group were measured in triplicate.

\section{Cell culture}

Human osteoblasts (BT-1008; Articular Engineering, Northbrook, IL, USA) from population numbers 2-6 were cultured in Dulbecco's modified Eagle medium (Gibco ${ }^{\circledR}$, Life Technologies, Carlsbad, CA, USA), supplemented with $10 \%$ fetal bovine serum (Life Technologies) and 1\% penicillin/ streptomycin (Life Technologies) under standard cell culture conditions (humidified, 5\% CO2/95\% air environment) at $37^{\circ} \mathrm{C}$. Prior to cell culture, frozen vials were thawed to $37^{\circ} \mathrm{C}$ and seeded in polystyrene tissue culture flasks (Corning Incorporated, Lowell, MA, USA) with $15 \mathrm{~mL}$ of culture media. Cell culture media was changed every 48 to 72 hours.

\section{Cell adhesion and proliferation}

Prior to the cell culture experiments, samples were sterilized in $70 \%$ ethanol for 10 minutes, washed twice with deionized water, and then dried in air at room temperature for 30 minutes. Each titanium alloy sample was seeded with $1 \times 10^{5}$ cells, and the media was changed every 48 to 72 hours. The same cell culture media that was used to expand the cells was also used for cell adhesion and proliferation assays. After the prescribed periods of 1,4 , and 7 days, the discs were rinsed in phosphate buffered saline (Life Technologies) to remove any nonadherent cells, and they were then moved to a new culture plate. The discs were then treated with $600 \mu \mathrm{L}$ of $0.05 \%$ trypsin-ethylenediaminetetraacetic acid (Life Technologies) for 10 minutes to remove all adherent cells from the titanium alloy surface. Cells were then stained with Trypan Blue (Sigma-Aldrich) and counted using an automatic cell counter (Auto T4 Cellometer; Nexcelom Bioscience LLC, Lawrence, MA, USA). Surfaces were analyzed to ensure that all cells were removed during this process.

\section{Long-term osteoblast functions}

Intracellular protein synthesis, alkaline phosphatase activity, and calcium deposits were measured at time points of 7 and 14 days to assess long-term osteoblast functions (all spectrophotometric kits were from Sigma-Aldrich). For this, each sample was seeded with $1 \times 10^{5}$ cells. Cells were cultured using the same cell culture media from the cell adhesion and proliferation studies (Dulbecco's modified Eagle Medium supplemented with $10 \%$ fetal bovine serum and $1 \%$ penicillin/streptomycin) with the addition of $10 \mathrm{mM}$ of $\beta$-glycerophosphate (Calbiochem, Merck Millipore) and $50 \mu \mathrm{g} / \mathrm{mL}$ L-ascorbic acid (Acros Organics, Thermo Fisher Scientific, Waltham, MA, USA) to facilitate the synthesis of calcium. Standard assays using commercial kit instructions were followed. ${ }^{10}$

\section{Statistics}

Numerical data was analyzed using standard analysis of variance techniques followed by a Student's $t$-test, and the statistical difference was considered to be significant at $P<0.05$. All experiments were completed in triplicate, and were repeated three times each.

\section{Results \\ Surface characterization}

The appearance of each group after treatment is displayed in Figure 1. The polished sample (Group A) showed visible striations, while all other samples showed a matte finish. As expected, the current commercially-used sulfuric acid anodized sample (Group C) changed to a green/yellow hue, while the hydrofluoric acid anodized sample (Group D) partially maintained the green/yellow hue, although it changed slightly. The hydrofluoric acid-etched sample (Group E) appeared to be slightly darker than the original bead blasted sample (Group B). Such results indicate that the anodization in hydrofluoric acid after anodizing in sulfuric acid can maintain (at least partially) a desirable color coding for identification purposes. Of note, the magnitude of the voltage was not changed in this study, but future studies should

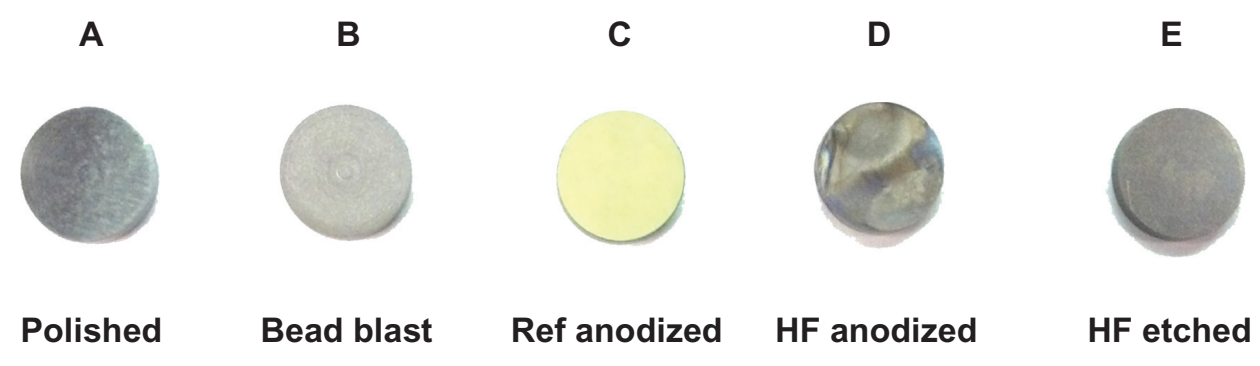

Figure I Change in surface appearance from the different treatment methods. Abbreviations: Ref, reference; HF, hydrofluoric acid. 
alter this important variable as it can drastically affect surface morphology.

Scanning electron microscopy images of each sample were captured and are shown in Figure 2. The polished samples (Group A) showed striations roughly $1-2 \mu \mathrm{m}$ in width. The bead blasted samples (Group B) showed the grain structure of Ti6Al4V. The sulfuric acid anodized samples (Group C) showed rounded contours that were $2 \mu \mathrm{m}$ in width. The hydrofluoric acid anodized samples (Group D) showed a pore size of 1-2 $\mu \mathrm{m}$ in diameter. Interestingly, such results indicate a significantly greater pore size when anodizing in hydrofluoric acid after anodizing in sulfuric acid (1-2 $\mu \mathrm{m}$ in diameter) than when anodizing in hydrofluoric acid alone, as reported previously (20-120 nm in diameter). ${ }^{5-9}$ Group E samples did not possess any nanotubular or nanoporous features, but they did display numerous nanometer rough features.

Contact angle measurements were recorded for each group (Table 2). The polished samples (Group A) had the highest contact angle at 83.4 degrees, while the hydrofluoric acid anodized (Group D) and the hydrofluoric acid-etched (Group E) samples had the lowest contact angles at 57.9 and 59.9 degrees, respectively. This indicates that the hydrofluoric acid anodized and hydrofluoric acid etched samples had a higher surface energy than the other sample groups.

Energy dispersive spectroscopy revealed the chemical composition of each sample at the surface, as shown in Table 3. The results revealed that the polished (Group A) and sulfuric acid anodized (Group C) samples both had small amounts of carbon on the surface. No fluorine was found on the surface of any of the sample groups. Both anodized samples showed the presence of oxygen, with the sulfuric acid anodized samples (Group C) having a higher percentage at $18.92 \%$ in comparison to the hydrofluoric acid anodized samples (Group D) at $6.11 \%$. All samples also showed trace amounts of iron present, ranging from $0.28 \%$ to $0.36 \%$. This may have been caused by remnants of the machining process that were not removed from passivation. All samples contained a lower amount of aluminum than was specified in the chemical composition sheet at 5.5\%-6.5\%. Additionally, each of the groups, except for the sulfuric acid anodized group (Group C, at 2.96\%) and the hydrofluoric acid anodized group (Group D, at 3.35\%) showed values within the specified range for vanadium, which was listed in the chemical composition sheet at $3.5 \%-4.5 \%$.

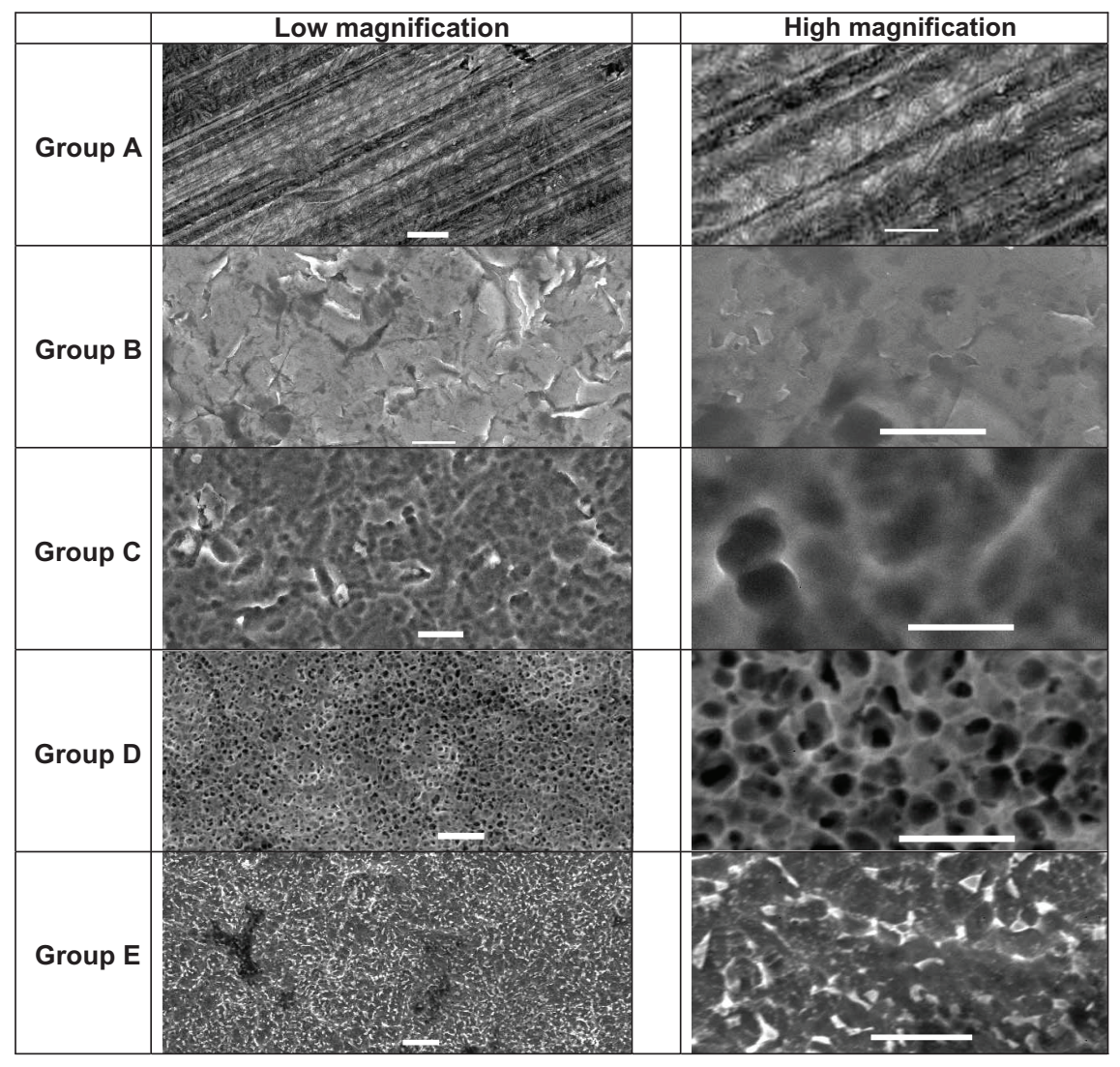

Figure 2 Scanning electron microscopy images of each sample.

Note: Scale bars on the left represent $10 \mu \mathrm{m}$ and scale bars on the right represent $5 \mu \mathrm{m}$. 
Table 2 Contact angle measurements

\begin{tabular}{lll}
\hline Groups & Theta $(\boldsymbol{\theta})$ & Standard deviation \\
\hline A - polished & 83.4 & 4.4 \\
B - bead blasted & 68.9 & 3.5 \\
C - sulfuric acid anodized & 72.2 & 3.9 \\
D - hydrofluoric acid anodized & 57.9 & 1.9 \\
E - hydrofluoric acid etched & 59.9 & 3.1 \\
\hline
\end{tabular}

\section{Osteoblast adhesion and proliferation}

The compiled results of osteoblast adhesion and cell proliferation experiments are shown in Figure 3. Most importantly, osteoblast numbers were greater for Group D than among all of the other groups studied here. Such results provided the first evidence that using hydrofluoric acid anodization after commercially-used sulfuric acid anodization improves osteoblast density (while retaining color coding, as previously demonstrated). Each group also demonstrated an increase in the number of adherent cells from 24 hours to 4 days, and from 4 days to 7 days.

\section{Alkaline phosphatase activity}

The alkaline phosphatase activity levels were measured after 7 and 14 days of osteoblast culture (Figure 4), and these results can be used as an indication of osteoblast differentiation. Similar to the osteoblast density results, the hydrofluoric acid anodized samples (Group D) had the highest alkaline phosphatase activity after both 7 and 14 days compared to any other samples tested here. Again, these results provided the first evidence that using hydrofluoric acid anodization after commercially-used sulfuric acid anodization improves osteoblast alkaline phosphatase activity, likely as a consequence of the greater number of initially adherent osteoblasts (while retaining color coding, as previously demonstrated).

\section{Osteoblast total protein content}

Total protein content was measured for each sample at 7 and 14 days. As displayed in Figure 5, the hydrofluoric acid anodized samples (Group D) had the highest average total protein content at both periods compared to all other samples.
Again, such results provided the first evidence that using hydrofluoric acid anodization after commercially-used sulfuric acid anodization improves osteoblast total protein content, likely as a consequence of the greater number of initially adherent osteoblasts (while retaining color coding, as previously demonstrated).

\section{Calcium deposition}

The amount of calcium deposited by the osteoblasts was measured on each sample at 7 and 14 days (Figure 6). After both periods (and similar to all of the previously reported results), the hydrofluoric acid anodized samples (Group D) had the highest level of calcium deposition compared to all other samples. Impressively, calcium deposition was three and two times greater when using hydrofluoric acid anodization after sulfuric acid anodization compared to using sulfuric acid anodization alone after 7 and 14 days, respectively. Again, these results provided the first evidence that using hydrofluoric acid anodization after commerciallyused sulfuric acid anodization improves osteoblast calcium deposition, probably as a consequence of the greater number of initially adherent osteoblasts (while retaining color coding, as previously demonstrated).

\section{Discussion}

The present study demonstrated, for the first time, significantly different surface properties resulting from the double anodization of Ti6Al4V with $0.8 \%$ sulfuric acid for 90 seconds at $96 \mathrm{~V}$, followed by anodization in $1.5 \%$ hydrofluoric acid for 5 minutes at $20 \mathrm{~V}$. The resulting surfaces possessed micron tubular structures that were notably larger than the nanotubular structures reported from using hydrofluoric acid alone, ranging from $15-120 \mathrm{~nm}$ in diameter. ${ }^{5-9,11}$

There are many possible reasons for this result, which will require additional experiments to accurately elucidate. As described by Macak et al, ${ }^{11}$ a key factor in controlling the tube diameters formed from anodizing titanium in hydrofluoric acid is the applied voltage. Titanium nanotube diameters have been achieved in the range of $15-120 \mathrm{~nm}$ for voltages

Table 3 Elemental concentration in the surface layer as determined by EDS

\begin{tabular}{|c|c|c|c|c|c|c|c|}
\hline \multirow[t]{2}{*}{ Samples } & \multirow[t]{2}{*}{ Description } & \multicolumn{6}{|c|}{ Elemental concentration in $\%$ weight } \\
\hline & & C & $\mathbf{0}$ & Al & $\mathbf{T i}$ & $\mathbf{V}$ & $\mathbf{F e}$ \\
\hline Group A & Polished & 4.65 & 0 & 4.75 & 86.76 & 3.56 & 0.28 \\
\hline Group B & Bead blasted & 0 & 0 & 5.18 & 90.61 & 3.85 & 0.36 \\
\hline Group C & Sulfuric acid anodized & 3.7 & 18.92 & 3.65 & 70.49 & 2.96 & 0.28 \\
\hline Group D & Hydrofluoric acid anodized & 0 & 6.11 & 4.86 & 85.33 & 3.35 & 0.35 \\
\hline Group E & Hydrofluoric acid etched & 0 & 0 & 4.82 & 90.83 & 4.07 & 0.28 \\
\hline
\end{tabular}

Abbreviations: EDS, energy dispersive spectroscopy; C, carbon; O, oxygen; $\mathrm{Al}$, aluminum; Ti, titanium; $\mathrm{V}$, vanadium; Fe, iron. 


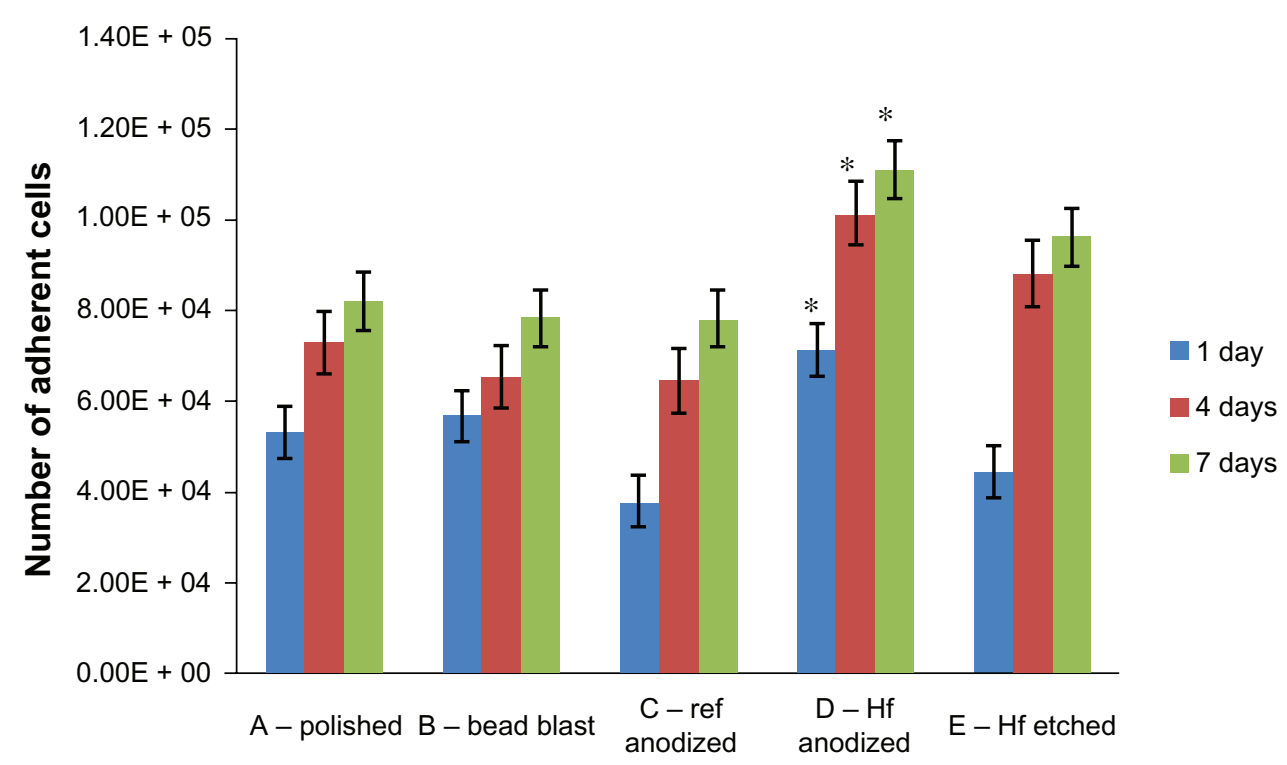

Figure 3 The number of adherent cells at all time points on the samples of interest to the present study.

Notes: Values are represented as the mean \pm SEM, $N=3$. $* P<0.01$ compared to all others at the same time period. For all samples, a significantly $(P<0.0 \mathrm{I})$ greater number of osteoblasts was found at increasing time periods.

Abbreviations: Ref, reference; HF, hydrofluoric acid; SEM, standard error of the mean; N, number.

varying between $1 \mathrm{~V}$ to $25 \mathrm{~V}$ when using hydrofluoric acid as an electrolyte. However, this does not explain the results observed in the current study, which used $20 \mathrm{~V}$ as an anodization voltage, and generated much greater diameter tubes on the micron scale. Since some materials in this study were previously anodized in sulfuric acid, this observed increase in nanotubular diameter could be a result of the already increased oxide layer formed from the initial anodization process.
Additionally, the pore diameter appears to be larger at approximately 5 to $7 \mu \mathrm{m}$; however, as shown for the first time here, even this larger tube diameter titanium alloy promoted osteoblast functions when compared to all other groups.

To explain this, we must remember that implant surface properties play a crucial role in mediating cell interactions. It is generally accepted that rough and porous surfaces have a more pronounced and beneficial influence on cellular activity

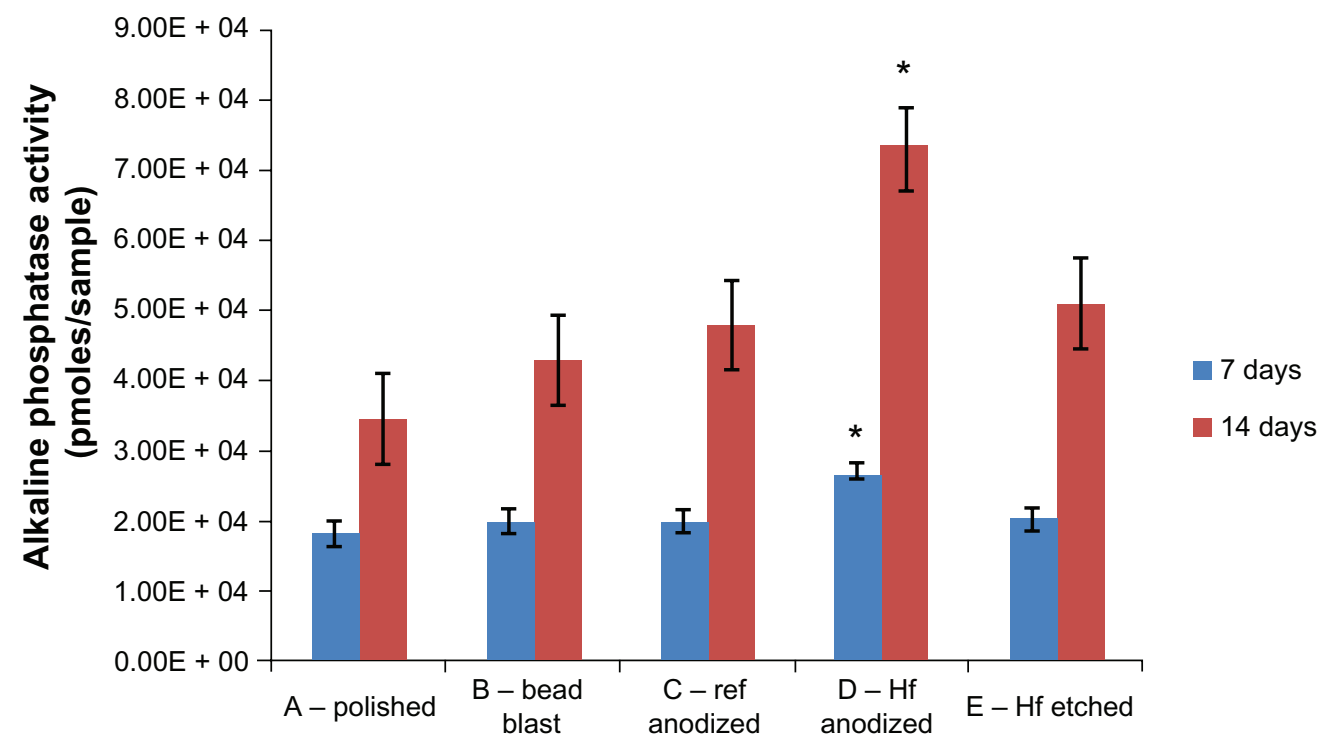

Figure 4 Alkaline phosphatase activity by osteoblasts cultured on the substrates of interest.

Notes: Values are represented as the mean $\pm \mathrm{SEM}, \mathrm{N}=3$. $* P<0.0 \mathrm{I}$ compared to all others at the same time period. For all samples, a significantly $(P<0.0 \mathrm{I})$ greater amount of alkaline phosphatase activity was found at increasing time periods.

Abbreviations: Ref, reference; HF, hydrofluoric acid; SEM, standard error of the mean; N, number. 


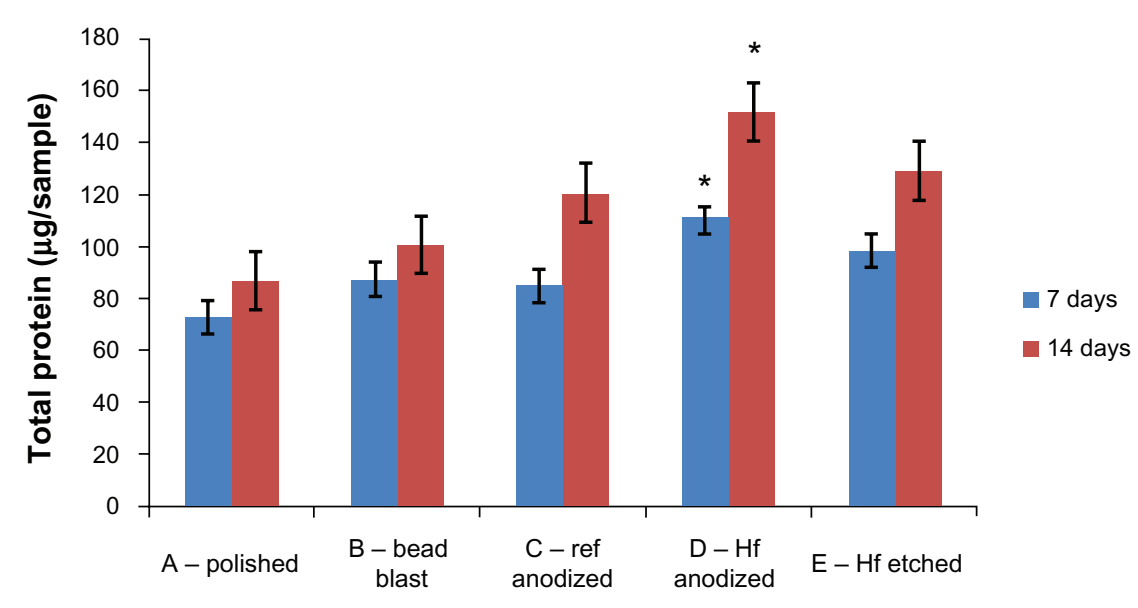

Figure 5 Total protein content of osteoblasts cultured on the substrates of interest to the present study.

Notes: Values are represented as the mean $\pm \mathrm{SEM}, \mathrm{N}=3$. $* P<0.01$ compared to all others at the same time period. For all samples, a significantly $(P<0.0 \mathrm{I})$ greater amount of total protein was found at increasing time periods.

Abbreviations: Ref, reference; HF, hydrofluoric acid; SEM, standard error of the mean; N, number.

than smooth surfaces. ${ }^{12}$ Surface topography is an important aspect of cell-material interactions, as it controls the surface area which influences net surface energy to mediate protein adsorption, which is important for anchorage-dependent cell functions..$^{13}$ The unique surface morphology of the samples treated in this study resulted in different osteoblast behavior. After anodizing the samples in hydrofluoric acid, osteoblast densities, alkaline phosphatase activity, and calcium deposition significantly increased in this study, demonstrating that such processes should be further investigated for orthopedic applications. The roughened appearance of the surface of Group D samples (sulfuric acid anodized, followed by hydrofluoric acid anodized) could provide a greater surface area for mediating more protein adsorption, which can foster more cellular focal adhesion than the smooth bead blasted or polished surfaces.

Another possible explanation for the increased osteoblast densities, alkaline phosphatase, and calcium deposition on the samples anodized first with sulfuric acid and secondly with hydrofluoric acid could be their increased hydrophilicity. The contact angle measurements in this study for the hydrofluoric acid anodized (Group D) and hydrofluoric acid-etched (Group E) samples were 57.9 and 59.9 degrees, respectively. This was significantly less than the polished samples (Group A) at 83.4 degrees, the bead blasted samples (Group B) at 68.9 degrees, and the sulfuric

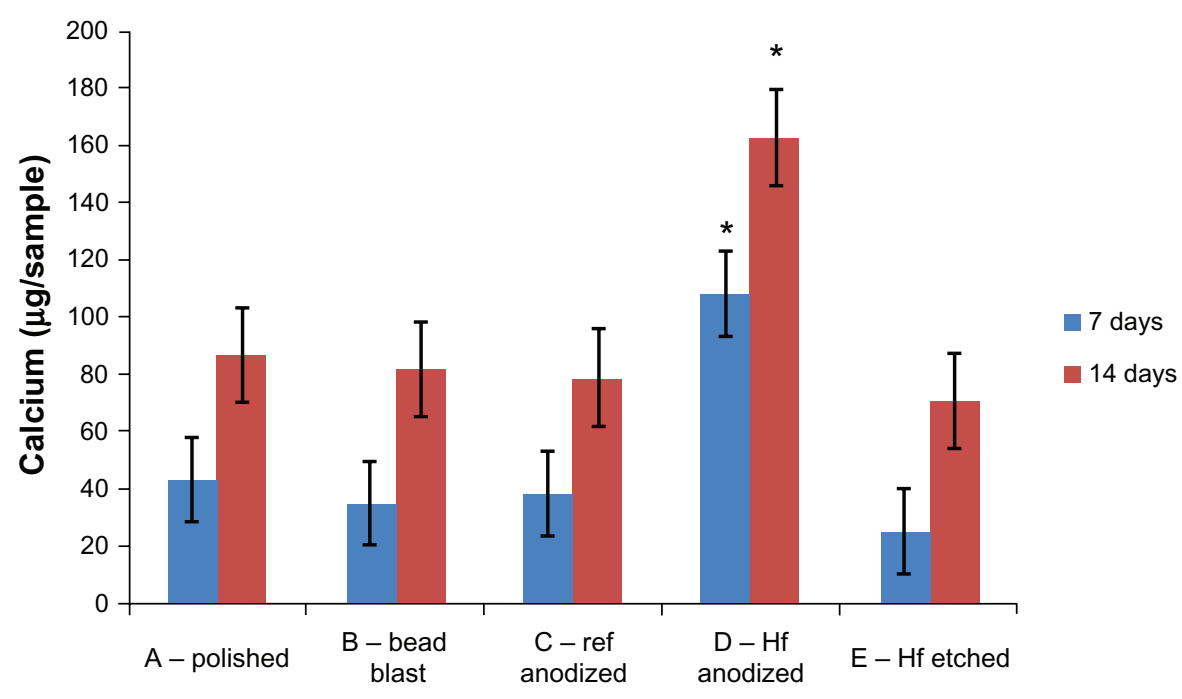

Figure 6 Calcium deposition by osteoblasts on the samples of interest to the present study.

Notes: Values are mean $\pm \mathrm{SEM}, \mathrm{N}=3$. $* P<0.0 \mathrm{I}$ compared to all others at the same time period. For all samples, a significantly $(P<0.0 \mathrm{I})$ greater amount of calcium was found at increasing time periods.

Abbreviations: SEM, standard error of the mean; N, number. 
acid anodized samples (Group C) at 72.2 degrees. A previous study has demonstrated that such increases in hydrophilicity of titanium-based alloys can lead to greater competitive adsorption of vitronectin, an important osteoblast adhesive protein. ${ }^{14}$

Importantly, this study correlated greater osteoblast densities to greater alkaline phosphatase and calcium deposition on Ti6Al4V that was first anodized in sulfuric acid and then anodized in hydrofluoric acid. Alkaline phosphatase activity is an early marker for osteoblast differentiation. Additionally, alkaline phosphatase activity has been shown to increase with increased surface roughness. ${ }^{10}$ This study showed that the samples treated with hydrofluoric acid (Group D) significantly improved alkaline phosphatase activity levels in comparison to all other samples at 1 week, and treatment with hydrofluoric acid significantly improved alkaline phosphatase activity in comparison to the polished (Group A), the bead blasted (Group B), and the sulfuric acid anodized samples (Group C) after 2 weeks of cell culture. This could be a result of the increased cell adhesion, which could lead to greater cell-to-cell contacts, and thus to greater alkaline phosphatase activity. These results correlate with those of other studies, which showed that titanium nanotubes promote osteoblast functions over conventional titanium surfaces. ${ }^{7}$

Lastly, the present study showed a significant increase in calcium deposition on the samples anodized in hydrofluoric acid (Group D) after 7 and 14 days. As the $\mathrm{TiO}_{2}$ layer forms, the Ti-OH groups on the surface act as nucleation sites for apatite crystals that favor deposition. The negatively charged $\mathrm{OH}$ interacts with the positively charged $\mathrm{Ca}^{2+}$ ions and causes a positive charge on the surface. This $\mathrm{Ca}^{2+}$ on the surface then interacts with phosphate groups to form amorphous calcium phosphate, which eventually crystallizes to bone-like apatite. Thus, in addition to mediating protein interactions in order to promote calcium deposition, by first anodizing Ti6Al4V in sulfuric acid and then anodizing it in hydrofluoric acid increases its surface area and consequently increases the net surface energy required to promote direct interactions with calcium - all while retaining the attractive color coding properties from the sulfuric acid anodization alone.

\section{Conclusion}

The present study demonstrated that the sequential anodization of Ti6Al4V using sulfuric acid followed by hydrofluoric acid produced unique microporous surface features approximately 1 to $2 \mu \mathrm{m}$ in diameter, and this promoted osteoblast densities, alkaline phosphatase activity, and calcium deposition while retaining the attractive color coding properties obtained from sulfuric acid anodization alone. Color coding is frequently used for titanium-based medical devices as a means to ease implantation, particularly for color coding different implant sizes. In this manner, this study provides the first evidence that color coded Ti6Al4V medical devices should be further anodized in hydrofluoric acid to improve osteoblast functions.

\section{Acknowledgments}

The authors acknowledge the financial support from DePuy Spine and the Hermann Foundation.

\section{Disclosure}

The authors report no conflicts of interest in this work. This study was conducted by a DePuy Spine employee. The samples in this study were provided by DePuy Spine.

\section{References}

1. Kruyt MC, van Gaalen SM, Oner FC, Verbout AJ, de Bruijn JD, Dhert WJ. Bone tissue engineering and spinal fusion: the potential of hybrid constructs by combining osteoprogenitor cells and scaffolds. Biomaterials. 2004;25(9):1463-1473.

2. Geetha M, Singh AK, Asokamani R, Gogia AK. Ti based biomaterials, the ultimate choice for orthopaedic implants - a review. Prog Mater Sci. 2009;54(3):397-425.

3. Cook SD, Salkeld SL, Stanley T, Faciane A, Miller SD. Biomechanical study of pedicle screw fixation in severely osteoporotic bone. Spine $J$. 2004;4(4):402-408.

4. Kienapfel H, Sprey C, Wilke A, Griss P. Implant fixation by bone ingrowth. J Arthroplasty. 1999;14(3):355-368.

5. Puckett SD, Taylor E, Raimondo T, Webster TJ. The relationship between the nanostructure of titanium surfaces and bacterial attachment. Biomaterials. 2010;31(4):706-713.

6. Ercan B, Webster TJ. Greater osteoblast proliferation on anodized nanotubular titanium upon electrical stimulation. Int J Nanomedicine. 2008;3(4):477-485.

7. Yao C, Webster TJ. Anodization: a promising nano-modification technique of titanium-based implants for orthopedic applications. In: Jackson MJ, Ahmed W, editors. Surface Engineered Surgical Tools and Devices. New York: Springer; 2007:21-47.

8. Yao C, Perla V, McKenzie JL, Slamovich EB, Webster TJ. Anodized Ti and Ti6Al4V possessing nanometer surface features enhance osteoblast adhesion. J Biomed Nanotechnol. 2005;1(1):68-73.

9. Rajyalakshmi A, Ercan B, Balasubramanian K, Webster TJ. Reduced adhesion of macrophages on anodized titanium with select nanotube surface features. Int J Nanomedicine. 2011;6:1765-1771.

10. Webster TJ, Ergun C, Doremus RH, Siegel RW, Bizios R. Enhanced functions of osteoblasts on nanophase ceramics. Biomaterials. 2000; 21(17): 1803-1810.

11. Macak JM, Tsuchiya $\mathrm{H}$, Ghicov $\mathrm{A}$, et al. $\mathrm{TiO}_{2}$ nanotubes: self-organized electrochemical formation, properties and applications. Current Opinion in Solid State and Materials Science. 2007;11(1-2):3-18.

12. Variola F, Yi JH, Richert L, Wuest JD, Rosei F, Nanci A. Tailoring the surface properties of Ti6A14V by controlled chemical oxidation. Biomaterials. 2008;29(10):1285-1298.

13. Das K, Bose S, Bandyopadhyay A. Surface modifications and cellmaterials interactions with anodized Ti. Acta Biomater. 2007;3(4): 573-585.

14. Webster TJ, Ergun C, Doremus RH, Siegel RW, Bizios R. Specific proteins mediate enhanced osteoblast adhesion on nanophase ceramics. J Biomed Mater Res. 2000;51(3):475-483. 
International Journal of Nanomedicine

Dovepress

\section{Publish your work in this journal}

The International Journal of Nanomedicine is an international, peerreviewed journal focusing on the application of nanotechnology in diagnostics, therapeutics, and drug delivery systems throughou the biomedical field. This journal is indexed on PubMed Central, MedLine, CAS, SciSearch ${ }^{\circledR}$, Current Contents ${ }^{\circledR} /$ Clinical Medicine,
Journal Citation Reports/Science Edition, EMBase, Scopus and the Elsevier Bibliographic databases. The manuscript management system is completely online and includes a very quick and fair peer-review system, which is all easy to use. Visit http://www.dovepress.com/ testimonials.php to read real quotes from published authors.

Submit your manuscript here: http://www.dovepress.com/international-journal-of-nanomedicine-journal 